\title{
Fuzzy-based Classification of Vaporous Compound using Electronic Nose
}

\author{
Rajina R. Mohamed, Azahar T. M, M A Mohamed, Rozita Ismail, RazaliYaacob
}

\begin{abstract}
The Fuzzy logic is a promising method in Artificial Intelligence. This is extensively employed to manage factors in the surroundings. The influence of fuzzy logic is demonstrated by evidence of making of proposed intelligent systems based on fuzzy logic methods e.g. pressure cooker, refrigerator, washing machine and so on. The intention of proposed work is to design an e-nose capable of detecting the distinct smell generated by various chemical vapours. These sensors of gas were used in this scheme. These readings from sensors passed to a fuzzy logic controller. The fuzzification process is taken by Mamdani style. The outcome of the proposed scheme is displayed at LCD display and matched to the outcome of simulation done using Matlab.
\end{abstract}

Keywords: fuzzy logic, Electrical Nose, Gas Sensors, vaporous compound.

\section{INTRODUCTION}

Humans have five great senses that can help them in evaluating surroundings. Eyes are used to assess the quality of seeing, ears are used to be sensitive to sound, skin sensitive towards touching, a mouth is significant to taste something and nose is used to evaluate the smell. The perception of the human nose idea is great in evaluating the quality of foods, cosmetics, chemicals et cetera. Thus, the technology using the proposed nose concept is created to assist humans in their life. This artificial nose is called electronic nose (e-nose) which is used as a sensor of gas to detect the gas molecules spread by 'product' which need to be analysed [1]. The similarity of an electrical nose and a biological nose is nothing but matching of a bee eye with an eye of a human. That's why, the correlation between human smell sensation and e-nose data gathering interpreted in the same manner. In different perspectives, the analysis of non smelly volatiles e.g. detection of explosives are also doable. So, the terminology of "electrical nose (e-nose)" creates an impression that we are going to make a nose that will look like the human nose. Till now, extensive research has been done to analyse the basics of smellant receptors and the structure of the smell sensory process. In every smell receptor cell, a specific kind of odorant receptor is placed, which is able to sense target smell. Whereas, complex smell which is made of various molecules required to activate different receptors. The final pattern of the receptor decides our sensation of the smell. Considering the technical limitations of the e-nose, we should characterize it as an effort to mimic the process of smelling. The gas sensor data

Revised Manuscript Received on September 14, 2019.

Rajina R. Mohamed, UniversitiTenagaNasional, Malaysia.(Email rajina@uniten.edu.my)

Azahar T. M, Universiti Kuala Lumpur, Malaysia.

M A Mohamed, Rozita Ismail, Universiti Sultan ZainalAbidin (UniSZA).(Email: rozita@uniten.edu.my)

RazaliYaacob, Universiti Putra Malaysia (UPM), Malaysia.(Email: razaliy@upm.edu.my) are processed to extract features in order to eliminate repetition and to reach at a description of the mixture of volatiles [2]. Fig. 1 is comparing the human nose to the enose.

\section{PROBLEM STATEMENT}

The problem behind the real application of e-noses is that the target gases belong to different characteristics features and mixed with contaminants like water vapour, etc. The gas sensors are able to detect those gases which are not detectable by the human nose. Humans have no receptors for water vapour. Similarly, we are not capable of detecting carbon monoxide (CO). Therefore, either detectable or not detectable gases and relevant and not relevant gases are two different research areas of e-nose research and development and need to describe in detail for the need of smell detection. Hence, we use both real-time approach and masssensitive approach of gaseous analyzers from diverse sources e.g. to detect the various smell generated by distinct chemical vapour. In the past, e-noses gained tremendous popularity in gas sensing. When an electrical nose imitates the mammalian sense of smell then it becomes more influential among scientists and researchers.Therefore, quartz crystal microbalance (QCM) based multi-sensor arrays is sensitive to different modules in order to produce a complex pattern recognition process [3]. Molecularly imprinted polymers on a multichannel mass transducer specify the receptor cells for an e-nose and pattern recognition process takes the role of mind to identify multiple parts e.g., monitoring smells originating from plants. These conceptualized artificial electronic processes are matchable with natural process, though with quite a various selectivity, gas sensor output patterns and proved their real-time applications. Therefore, we analyze a methodology that has utilization in multiple industries such as food processing and quality assurance.

\section{METHODOLOGY}

\subsection{E-Nose Concept}

Design of a device for electronic smell sensory is an appealing technology in the field of chemical sensors. In the other research areas of taste sensing, sensing in liquid phase is possible with the use of electronic tongues (e-tongues). In the other words, e-nose is designed for detecting gases, e.g. smell, aroma, odor etc. These instruments are replicating the living smell process with the help of multivariate data investigation tools. Increasing interest of researchers in 
environment, security, food quality control and health also make a way to develop technology of chemical sensor e.g. e-nose. Researchers have been doing research since 1970. In the past, Infrared Spectroscopy (IRS), Fourier Transform Infrared Spectroscopy and Mass Spectrometry of Gas Chromatography, Nuclear Magnetic Resonance (NMR) were in trends for chemical investigation [1]. The most famous prototype of an electrical nose was developed by Persaud and Dodd in 1982 [4]. Their concept was detection of volatile compounds generated by microorganisms. They researched various stages of the human smell sensory process and measured volatile compounds [2]. Signal monitoring, amplification were measured by apparatus whereas neural networks are used for pattern recognition. Both e-noses and chemical based sensor technology helped humanity in different areas: diagnosis of human diseases, quality control in the food sector etc. Gardner and Bartlett in 1994 [5] characterized an electrical nose as: an device consisting of multiple channels of chemical based sensors with partial specificity and an efficient model for pattern detection [4] for recognition of simple or complex mixtures. Fig. 1 demonstrates the similarity of the human system and and electrical nose.

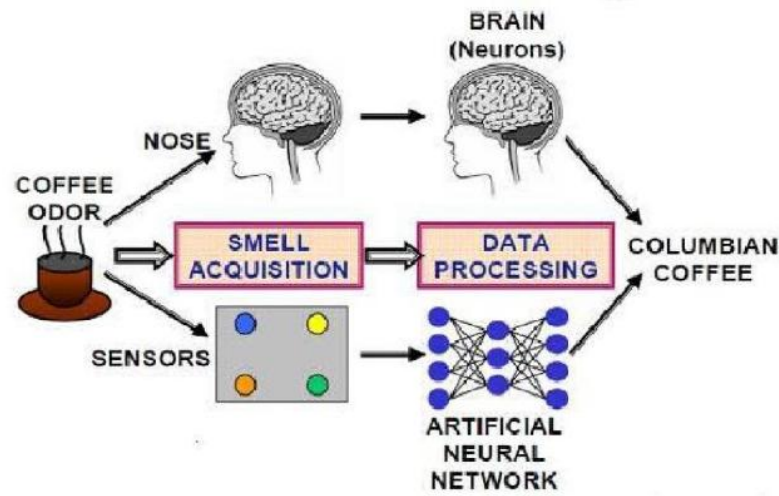

Fig. 1: Comparing the Human Nose to the Electrical Nose

The basic parts of an electrical nose is:

- A specimen handling methodology.

- A recognition methodology based on a general approach.

- A methodology of data science, e.g., various tools made for Pattern recognition.

A detection methodology contains a number of gas sensors to detect gases generated by vaporous compounds. The sensor of gas computes the presence of gas in the surrounding. The sensor of gas deals with a gas to determine its presence. Each gas has a unique feature after computing voltages. The presence of the gas is decided by computing the current discharge. There are different utilization of gas sensors like process control, monitor environment, controlling boiler, detection of fire, breath test of alcoholic person, mines safety, home safety and grading of agriculture products e.g. coffee and spices. They are six gas sensing technologies, i.e. Gas Sensor using Metal Oxide, Capacitance, Acoustic Wave, Calorimetric, Optical and Electrochemical characteristics[6]

\subsection{Fuzzy Logic Classification}

LoftiZadeh gave an evolutionary Fuzzy set theory in 1965. It was developed to define uncertainty and vagueness with the help of formalized logical tools. It deals with the imprecision inherent in various real-life situations [7]. Till then, Bivalent set theory dominated western logic. Whereas, statements were either true i.e. 1 or false i.e 0 . In a set, things either were a member or not a member. The powerful characteristics feature of bivalent sets was mutual exclusivity. Mamdani and sugeno fuzzy logic are two kinds of common fuzzy logic used. Basically for students, mamdani is the preferred type because it is easier to understand [8].

\subsection{Principles of data fusion}

'Data' is information that can come from one or various sources while 'Data Fusion' is the process of combining multiple data from different sources in order to generate more meaning to the user. Data fusion processes are classified as low, intermediate or high [9]. Two powerful components of an e-nose; sensing methodology and automated pattern recognition methodology. The limitation in Electrical Nose can be minimized by optimizing the sensor array and the artificial intelligence process [10]. Nowadays, the complexity of the sensing environment data has increased. Using single pattern recognition might not be sufficient and give an effective result. Data fusion approach overcomes this limitation by integrating the data extracted by various patterns of recognition method [11]. Based on the previous research, there are three major structures of data fusion used: pre-processing, feature and decision level fusion. The moves of the system are described below.

- Pre-processing data fusion (raw data set is processed using various processing methodology and then integrated into a raw data set.)

- $\quad$ Feature fusion (Used numerous feature extraction methods to create the diverse feature vectors in various feature space)

- Decision level fusion (data mining with independent classifier. Then, a decision is taken by integrationof the decision of each classifier).

The common idea of multisensor data fusion is similar to the behaviour of humans and animals based on experience and reasoning to improve their probability of survival. The general challenge of data fusion from multiple sensors is to determine the best procedure for combining the reading of multiple sensor [12] . The favour of a statistical approach is that explicit probabilistic models are used to analyze the various associations [13].

\section{IMPLEMENTATION OF DESIGN}

\subsection{Hardware Design}

Hardware design contains a few components, i.e voltage regulator, both gas and chemical sensors and liquid crystal display (LCD). Voltage regulators frequently generate required power supply otherwise raw current destroy the 
circuit. The scope of a voltage regulator in a circuit is used to control voltage upto a desired value. The voltage regulator is needed for the sensor where it includes supply 5V for sensor MQ3 and TGS 2611 and 6V for MG811. While using test outcomes for analyses voltage, voltage for MG811 is equal to $0 \mathrm{~V}$ for all the testing. We don't know what the problem is, whether the sensor is destroyed or the value is very low. So, after discussion with the supervisor, we changed the sensor MG811 to TGS 800. With it, the entire sensor desired supply 5V. LCD displays come in different sizes most often named by the number of rows and then the length of the display line. For example a 1x16 LCD display will have one row of sixteen characters and a 4x20 LCD display will have four rows with twenty characters in each. Most LCDs that are being made now come with one row of sixteen pins. Display control is managed by fourteen pins. Backlighting is managed by two pins. In part of selecting the sensor, these kinds of the sensor are used which are MQ3, TGS 2611 and MG 800 for gas detecting.

\subsection{Characteristics of Sensor output}

The output of the sensor is characterized by association between the gas presence changes and resistance of the sensor changes. This association is based on algorithmic function. The output of the sensor is demonstrated in Fig. 2. In these figures, the resistance of the sensor values (Rs) are normalized according to the resistance of the sensor conditions (Ro) for each model, and the Y-axis is indicated as resistance of the sensor ratio: Rs/Ro. All the sensor characteristics represent typical characteristics.
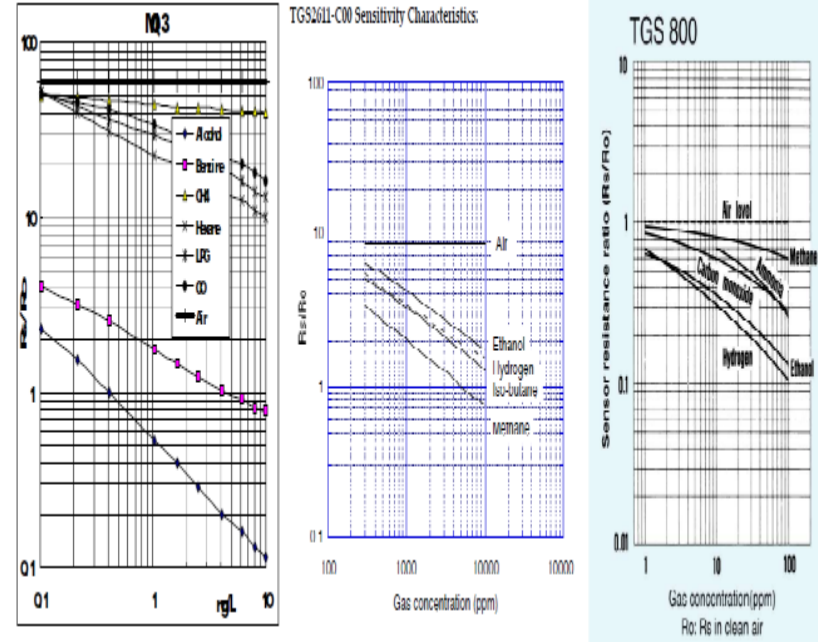

Fig. 2: Sensitivity of MQ3, TGS 2611 and TGS 800 sensor

In this work, proteus 7.5 are used for designing the voltage regulator and transformer. Fig. 3 is a prototype design used for testing and also demonstrated the connection of circuit. and also show design PCB layout.

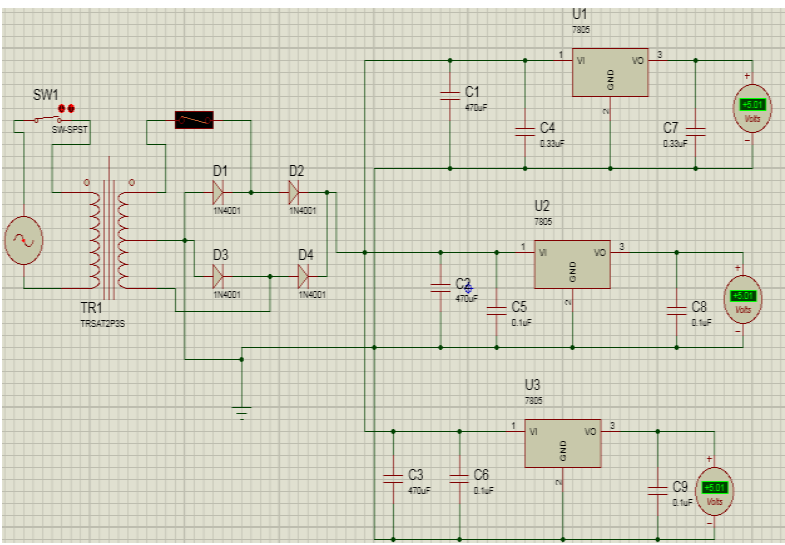

Fig. 3: PCB Layout of Voltage Regulator using PROTEUS

\section{RESULTS AND DISCUSSION}

Fig. 4 shows the complete scheme prototype contains the three sensors which detect gases from vaporous samples. Fig. 5 displays LCD display to record input and output.

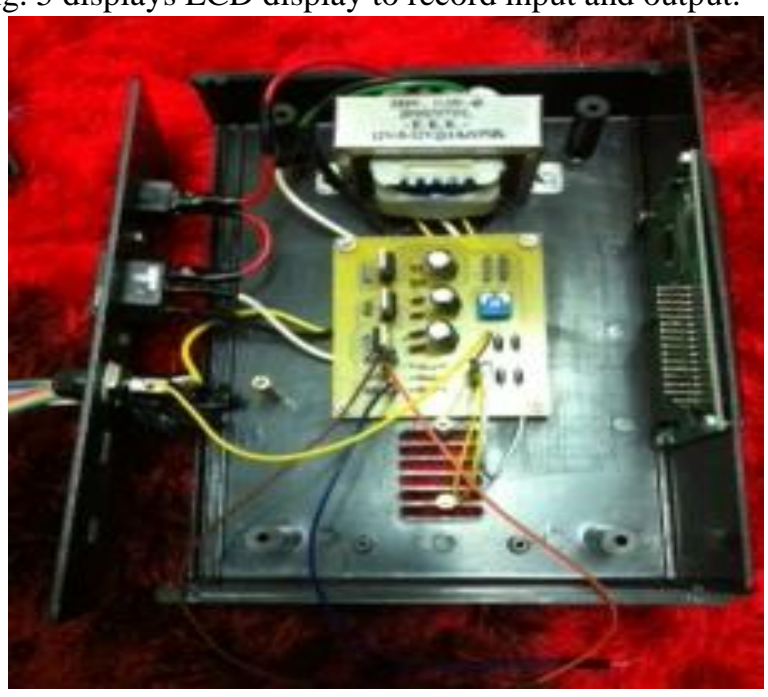

Fig. 4 : Design Prototype

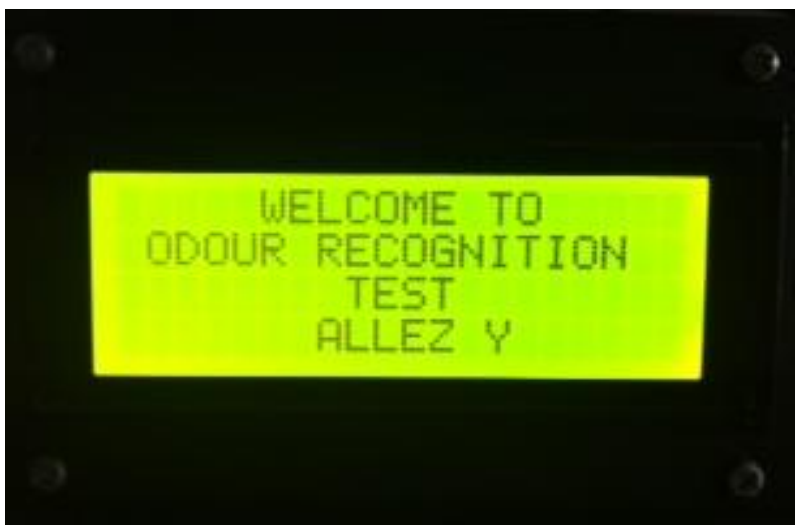

Fig.5 : LCD starting display.

Testing has been done in order to see the functionality and process of the designed device towards vaporous compounds. The sample of vaporous compounds in these works are coke, apple cider and perfume. Fig. 6 and 7 manifests the samples used in this design testing.

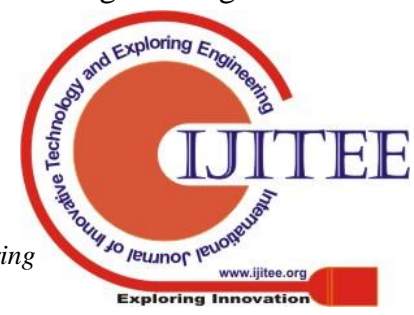




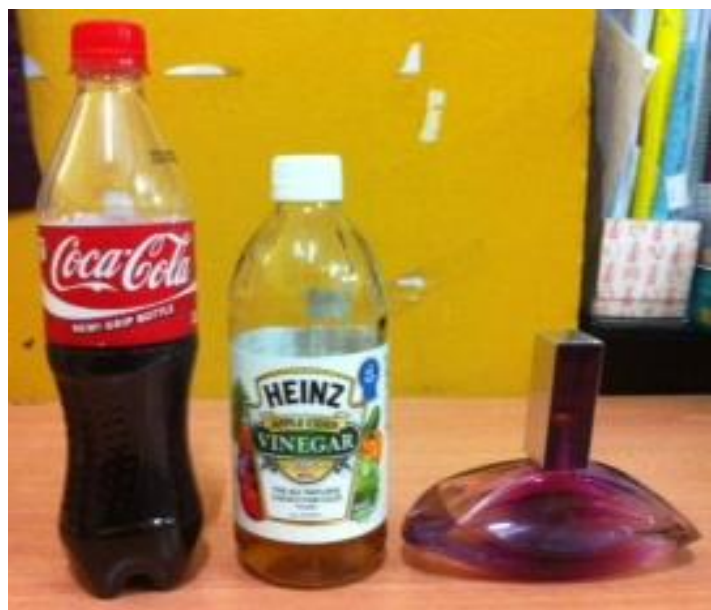

Fig.6 : Liquids for analysis testing.
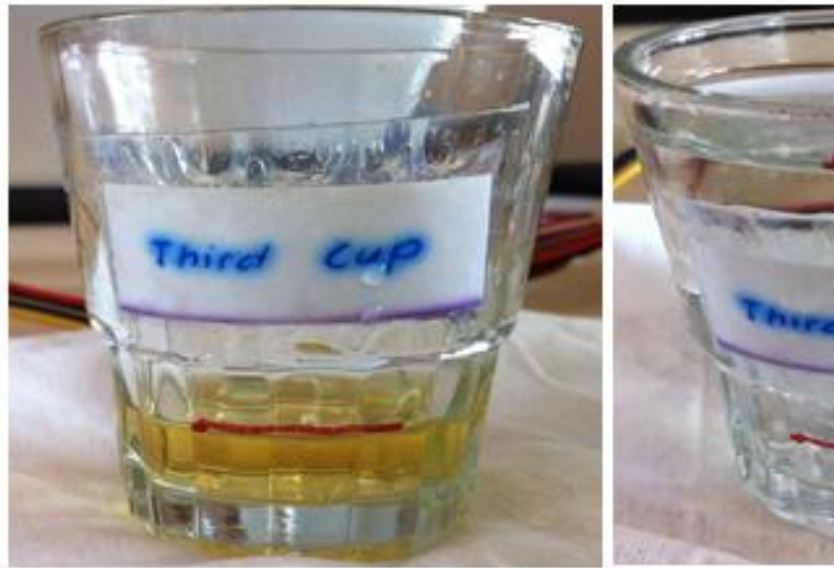

Fig. 7: Quantity of liquids for testing and Quantity of water for testing.

\section{1 Testing}

\subsubsection{Investigation of sample}

All specimens (Coke, Cider, perfume) have been tested with the help of sensor (MQ3, TGS2611 and TGS 800) by computing the sensor output simultaneously. The output is feeded to Arduino and the output is shown in serial monitoring for an interval of 30 seconds. The figure below is the outcome for each sensor. Fig. 8, 9, 10 illustrate the response of voltage when we tested with a sample with various computations as stated in each figure.
COKE TEST FOR TGS 800

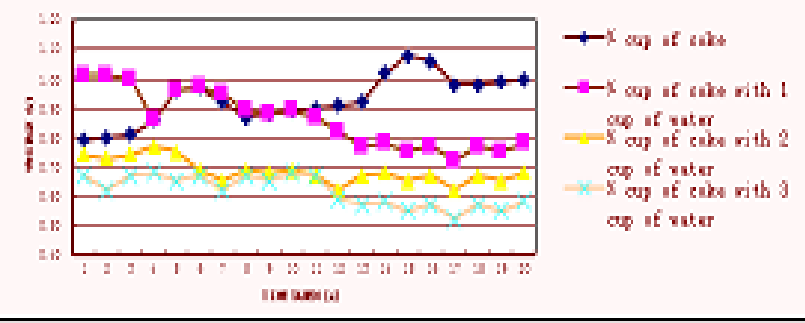

COKE TEST FOR TGS 2611
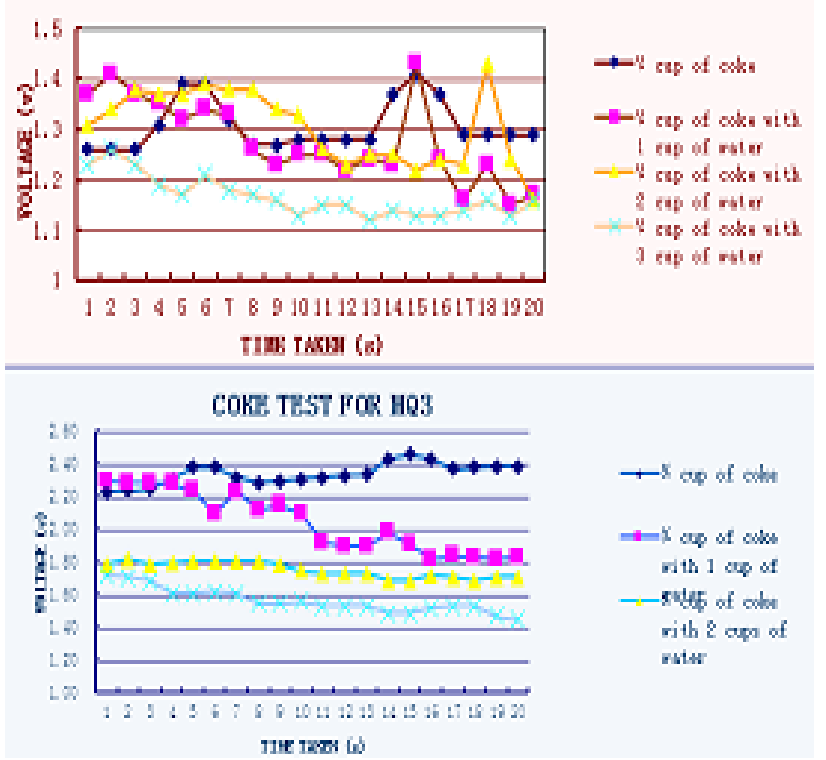

Fig. 8: Graph plotted for coke analysis using three various sensors.

APPLE CIDER TEST FOR TGS 800

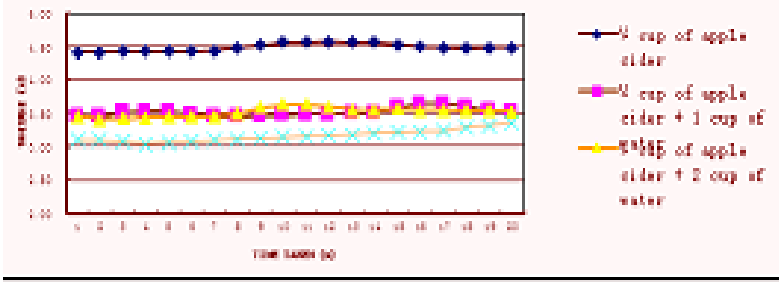

APPLE CIDER TEST FOR TGS 2611
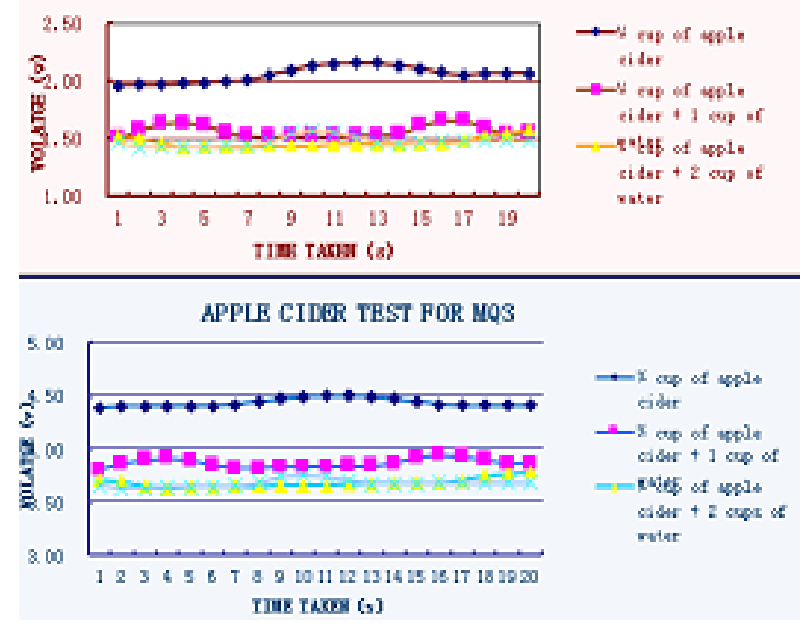

Fig. 9: Smell analysis of apple cider for three sensors

\section{Published By:}

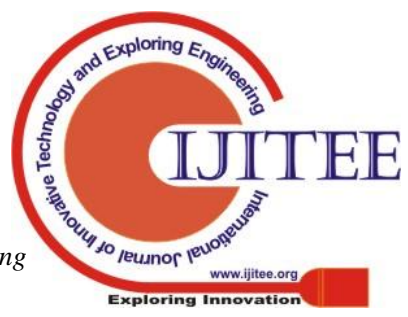


PERPLME TEST FOR TGS 800

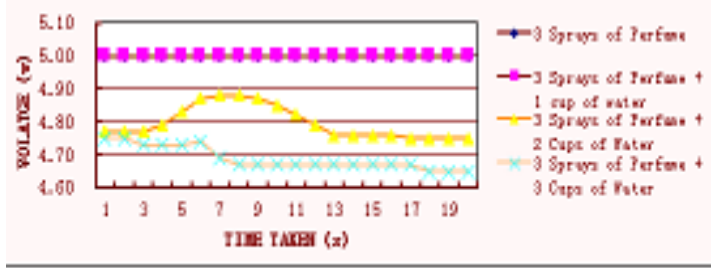

PERFLNE TEST FOR TGS 2611
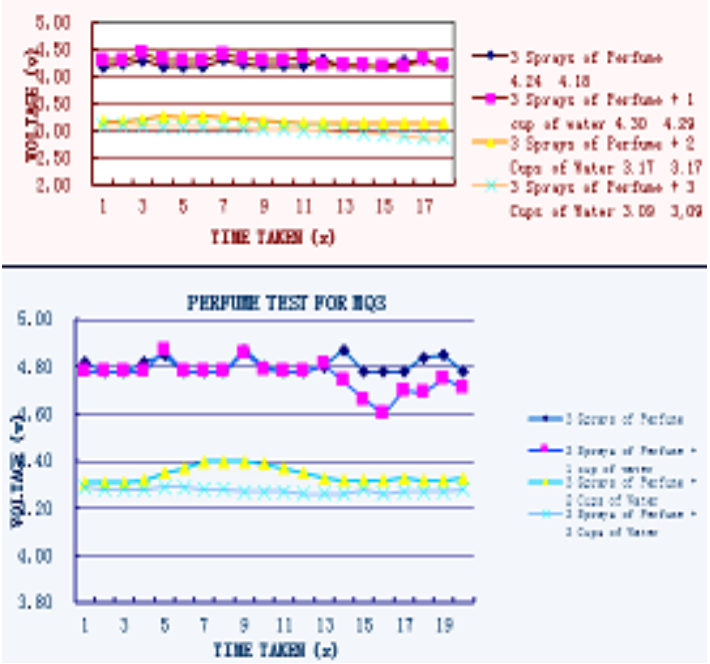

Fig. 10: Determination of perfume with three sensors

\subsection{Classification}

Gas classification is the second part in the Electrical nose and we used fuzzy logic to classify gases generated by all the vaporous samples. Fuzzy rules and fuzzy inference processes are characterized based on three numbers of sensors and combined by AND operations. The stages of the fuzzy logic classifier are Fuzzification, Fuzzy inference methodologies and Defuzzification [17]. Twenty-seven rules are designed in order to classify gases from specimens. The rules created are based on the various scenarios of the linguistic variables. Fig. 11 demonstrates the example of fuzzy rules creation for the specimens and sensor values of gas.

IF (MQ-3 is Low) AND (TGS-2611 is Low) AND (TGS-800 is Low) THEN (Result is Coke)

IF (MQ-3 is Low) AND (TGS-2611 is Low) AND (TGS-800 is Medium) THEN (Result is Coke)

IF (MQ-3 is Low) AND (TGS-2611 is Low) AND (TGS-800 is High) THEN (Result is Coke)

IF (MQ-3 is Low) AND (TGS-2611 is Medium) AND (TGS800 is Low) THEN (Result is Coke)

IF (MQ-3 is Low) AND (TGS-2611 is Medium) AND (TGS800 is Medium) THEN (Result is Apple Cider)

IF (MQ-3 is Low) AND (TGS-2611 is Medium) AND (TGS800 is High) THEN (Result is Apple Cider)

IF (MQ-3 is Low) AND (TGS-2611 is High) AND (TGS-800

IF (MQ-3 is Low) AND (TGS-2611 is High) AND (TGS-800 is Medium) THEN (Result is Coke)

IF (MQ-3 is Low) AND (TGS-2611 is High) AND (TGS-800 is High) THEN (Result is Perfume)

IF (MQ-3 is Medium) AND (TGS-261 1 is Low) AND (TGS-

800 is Low) THEN (Result is Coke)

800 is Medium) THEN (Result is Apple Cider)

IF (MQ-3 is Medium) AND (TGS-2611 is Low) AND (TGS-

800 is High) then (Result is Apple Cider)

Fig. 11. The example of fuzzy rules creation is Low) THEN (Result is Coke)

IF (MQ-3 is Medium) AND (TGS-2611 is Low) AND (TGS-

Defuzzification values are required to decide the vapour intensity level of a sample. In this design, defuzzification is carried out using centroid method [15]. Fig. 12 demonstrates the outcome of this defuzzification. Defuzzification is performed using the centroid method and this defuzzification value can then decide the vapour level of a sample.

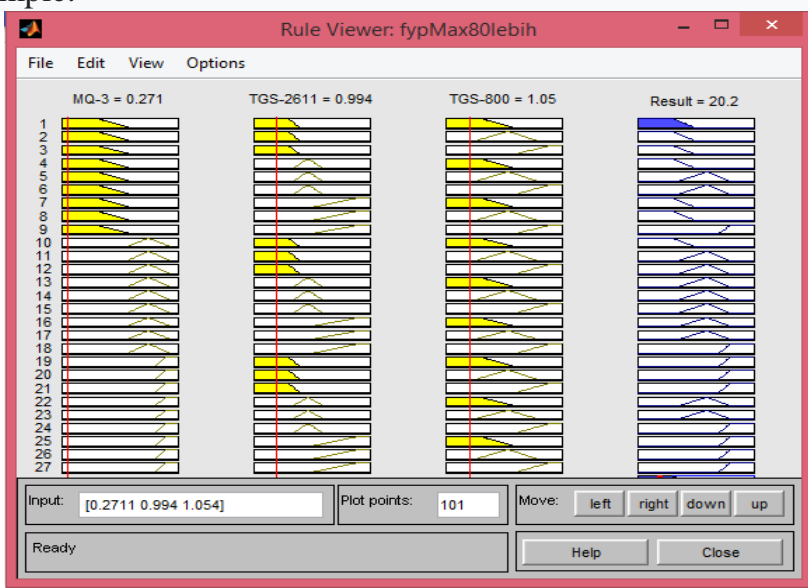

Fig. 12: The example of fuzzification result

Fuzzy Logic permits the proposed design to receive optimaloutcomes. The designed e-nose generates redundant responses in the computation of three liquids using varioussensors byconfirming its characteristics of reproducibility. The designed e-nose is capableof generatingdistinct patterns for distinctspecimens. Depending on the outcomes, we declare this electronic nose as a reliable analytical tool.

\section{CONCLUSION}

The objective of the design was to design our own electronic nose to decide the vapour quantity of a food item. A suitable sensor was selected and tests were performed to see if the device was capable of responding to the sample successfully, hence to determine its discriminative ability, reproducibility and repeatability[13]. Examining the quantity of vapour compounds is key to ensure the safety of the environment, especially fuel-sensitive. This test can be continued with some other chemicals that are more sensitive to human health.

\section{ACKNOWLEDGMENT}

This research was supported by FRGS 2018 Project code 20180101FRGS.

\section{REFERENCES}

1 Siong Fong Sim et al., "Synchronized Analysis of FTIR Spectra and GCMS Chromatograms for Evaluation of the Thermally Degraded Vegetable Oils', Journal of Analytical Methods in Chemistry, Volume 2014,

2 L. Carmel, et.al. "An eNose algorithm for identifying chemicals and determining their concentration", Sensors and Actuators B: Chemical, available online at www.sciencedirect.com.

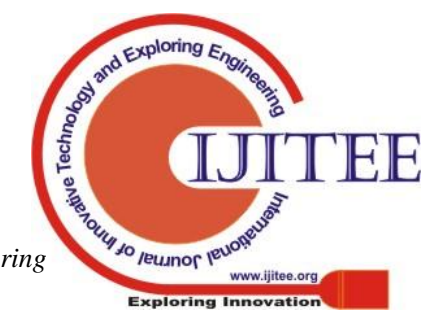


3 Bekir MUMYAKMAZ, Kerim KARABACAK, "An ENose-based indoor air quality monitoring system: prediction of combustible and toxic gas concentrations", Turkish Journal of Electrical Engineering \& Computer Sciences, 2015.

4 SigeruOmatu, Hedeo Araki, “Intelligent Classification of Odor Data Using Neural Network", 6th Intl Conf on Advanced Engineering Computing and Applications in Sciences, (ADVCOMP 2012), 2012.

5 A. Nopwinyuwong et al., " Development of a food spoilage indicator for monitoring freshness of skinless chicken breast", Talanta, july 2014.

6 Aleixandre M., et.al., Analysis of neural networks and analysis of feature selection with genetic algorithm to discriminate among pollutant gas. Sens. Actutaors B: Chem. 2004, 103, 122-128.

7 Md. MizanurRahman, et. al. " Classification and pattern recognition algorithms applied to E- Nose", Proceedings of International Conference on Electrical Information and Communication Technology (EICT 2015).

8 Jayeeta Sharma, AmlanJyotiBhagawati, RikuChutia, "Fuzzy Logic based Odour Classification System in Electronic Nose", International, 2013

9 M. Fatih Adak, NejatYumusak, "Classification of ENose Aroma Data of Four Fruit Types by ABC-Based Neural Network", Sensors 2016.

10 N. Alavi, "Quality determination of Mozafati dates using Mamdani fuzzy inference system", Journal of the Saudi Society of Agricultural Sciences, 2013

11 PushkarGoel, et.al. "Food Quality Assessment Using Fuzzy Logic", Proceedings of the $9^{\text {th }}$ INDIACom; INDIACom 2015.

12 MazlinaMamatet.al., " An Electronic Nose for Reliable Measurement and Correct Classification of Beverages", Sensors 2012.

13 P. Corcoran and H. Shurmer. An intelligent gas sensor. Sensor and Actuator A: Physical, 41-42:192-197, 1994

14 Azahar T. M., et.al. "A.Development of Fuzzy Logic Based Odor Detection", Journal of Science \& Engineering Technology JSET Vol: 02 No: 02, 2015

\section{AUTHOR PROFILES}

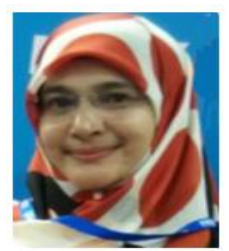

Rajina R. Mohamed received her bachelor degree in computer science from university of putra (upm), malaysia. she then pursued her master study in computer science filed majoring in distributed computing at the same university, completed her study in 2003. she is currently works as a lecturer in uniten, kualalumpur, malaysia since september 2013. previously, she was a researcher in mimosberhad focusing on computer networking, network information security, wireless communication (ipv4, ipv6), digital home and e-learning. currently doing research in artificial intelligence, iot and sensorbased application . she has published several publications and filed several patents on her research findings.

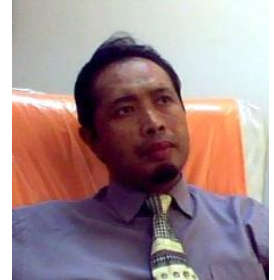

Dr. TengkuMohdAzahar received his Bachelor degree in Electrical and Informatic from Université de Nancy I, Nancy, France, in 1992, followed by his Master degree (Education) from UniversitiTeknologi Malaysia (UTM), Johor, Malaysia, in 2000, under the supervision of Professor Widad Othman. Finally, he obtained his Ph.D. degree (Computer Science) from Université de La Rochelle, La Rochelle, France, in 2013, under the supervision of Professor Patrice Boursier. He started his career as an Engineer at Intel Technology (M) Sdn. Bhd., Penang, Malaysia, in 2002. His career as a Lecturer started in 1996 at the Department of Industrial Automation, Malaysia France Institute, Bangi, Malaysia. In 2003, he joined Universiti Kuala Lumpur as a Senior Lecturer. Now, TengkuMohdAzahar is holding an Associate Professor position at the Department Industrial Automation, Universiti Kuala Lumpur Malaysia France Institute (UniKL MFI), since 2015. Since 2018, he serves as the Deputy Dean for Academic and Technology at Universiti Kuala Lumpur Malaysia France Institute (UniKL MFI).

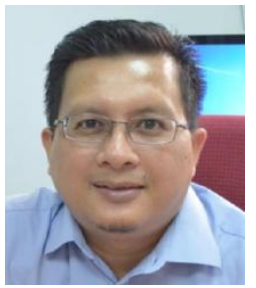

Assoc. Prof. RazaliYaakob received the Bachelor Degree in Computer Science in 1996 and Master in Computer Science from in 1999, from Universiti Putra Malaysia, and PhD from University of Nottingham, United Kingdom in 2008. Currently, he is a lecturer at the Faculty of Computer Science and IT, Universiti Putra Malaysia. His research areas include artificial neural network, pattern recognition, and evolutionary computation in game playing. $\mathrm{He}$ is a member of the Intelligent Computing Group at the faculty.

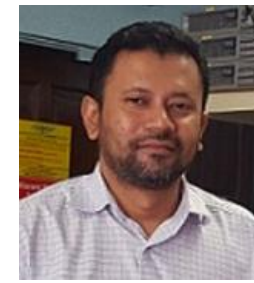

MohamadAfendee

Mohamed

received his $\mathrm{PhD}$ in Mathematica Cryptography in 2011 and currently serves as an associate professor at Universiti Sultan ZainalAbidin. His research interests include both theoretical and application issues in the domain of data security, and mobile and wireless networking. 


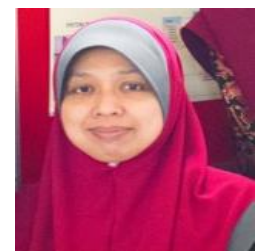

Rozita Ismail is currently a senior lecturer in the Department of Informatics, College of Computer and Information Technology, UNITEN. She has obtained her $\mathrm{PhD}$ in Information Technology in 2016. Her research interest includes Human-Computer Interaction/UX, Interface Design, Dyslexia and UAV. She has published over 30 publications including journals and international proceedings indexed by Scopus.

She can be reached at: irozita@uniten.edu.my 